\title{
COMPARISON: SIMULATION AND EXPERIMENTAL CHARACTERISATION OF AN ALL-OPTICAL GAIN- CLAMPED ERBIUM-DOPED FIBRE AMPLIFIER
}

\author{
T. Subramaniam, M. A. Mahdi, P. Poopalan, and H. Ahmad \\ Photonics Laboratory, Department of Physics, University of Malaya \\ 50603 Kuala Lumpur, Malaysia.
}

E-mail: sth_34@yahoo.com

\begin{abstract}
This paper presents the GC-EDFA characteristics comparison between the simulated results (using EDFA_Design software) and experimental results. The comparisons reveal the usefulness of the software in simulating the behaviour of an alloptical GC-EDFA system. Comparisons are made for values obtained from the system operated at high laser power, in order to highlight the differences between the experimental and simulated values. The main objective for this comparison is to prove the capability of the software in simulating the gain-clamped system. Therefore, the software can be used to test new configurations, aimed at improvising current gainclamped EDFA performances.
\end{abstract}

Key words: optical, gain-clamping, erbium, fibre amplifier, simulation, experiment

\section{INTRODUCTION}

The all-optical gain-clamped erbium-doped fibre amplifier was first introduced in 1991 by M. Zirngibl [1]. It is designed to solve the slow and undesirable gain fluctuations due to saturation effects in EDFA that occurs when the EDFA is used in networking and switch applications involving on/off-keyed packets or a multiplicity of WDM channels that are randomly turned on and off. The all-optical gain-clamped erbium-doped fibre amplifier is an erbium-doped fibre amplifier (EDFA) designed with a cavity (either ring or Fabry-Perot type). This device generates laser by utilising the amplified spontaneous emission (ASE) within the system in order to maintain the amplifier gain at a fixed value, independent of the input signal power. This feature also known as the gain linearization, is described by

$$
\frac{P_{s}^{\text {out }}}{P_{s}^{\text {in }}}=G=\text { constant }
$$

This holds for any input signal power falling in a given dynamic range [2]. The GCEDFA based on ring type cavities are more favourable, since the Fabry-Perot cavity is subject to; (a) spatial hole burning, causing inhomogeneous depletion of inversion in the laser medium and (b) strong mode competitions thus, is less stable [3].

An interesting ring-based GC-EDFA configuration is the counter-propagating GC-EDFA, which is proposed by Kobayashi and Muro [4]. It features elimination of laser from at the 
amplifier's output. This set-up is configured in such a way that the circulation of the lasing signal is in the opposite direction of the input signals, thus preventing the laser from being emitted at the system's output end. The design is shown below in Fig. 1.

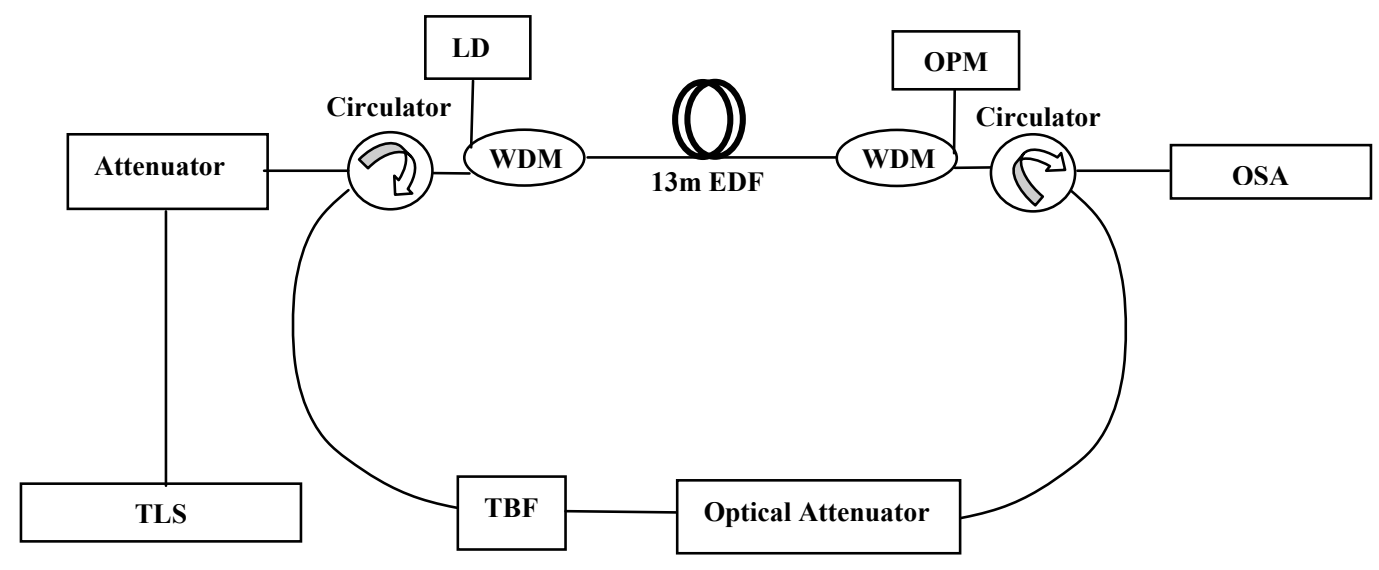

Fig. 1: Counter-propagating GC-EDFA configuration

This paper presents for the first time, comparison between GC-EDFA characteristic values based simulations using the EDFA_Design version 2.0 and actual experimental values. The EDFA_Design from Optiwave Corporation is one of the programs for basic EDFA design and offers accurate algorithms for simulations. It is designed to model the physical parameters of the set-up easily [5]. Earlier, this software was tested and evaluated for standard EDFA performances in the laboratory. The software accurately predicts the gain, noise figure and gain spectrum profiles accurately. The accuracy is much higher in the high pump and low signal region, less than 3\% discrepancy. Following this success, the software was then used to simulate the GC-EDFA system. The accuracy of these simulations is discussed below by comparing the simulation results with actual experimental values. Comparisons are made for values very high laser power, with clamped gain value of about $12 \mathrm{~dB}$. The strong clamping effect will highlight any obvious discrepancies between the experimental and simulated values.

\section{RESULTS AND DISCUSSION}

The comparisons between the simulated results and the experimental results displayed below include the gain and noise figure performances, the gain spectrum and the amplifier output profile. These comparisons are made in an effort to validate the capability of the EDFA_Design software in simulating results for a GC-EDFA system. As a result, this software can be further used to test new designs of the gain-clamped system aimed towards designing an optimized GC-EDFA system. Optimized GC-EDFA should display sufficiently high clamped gain with high critical input power, low noise figure and does not exhibit laser at the output end. 


\section{A. GC-EDFA Gain}

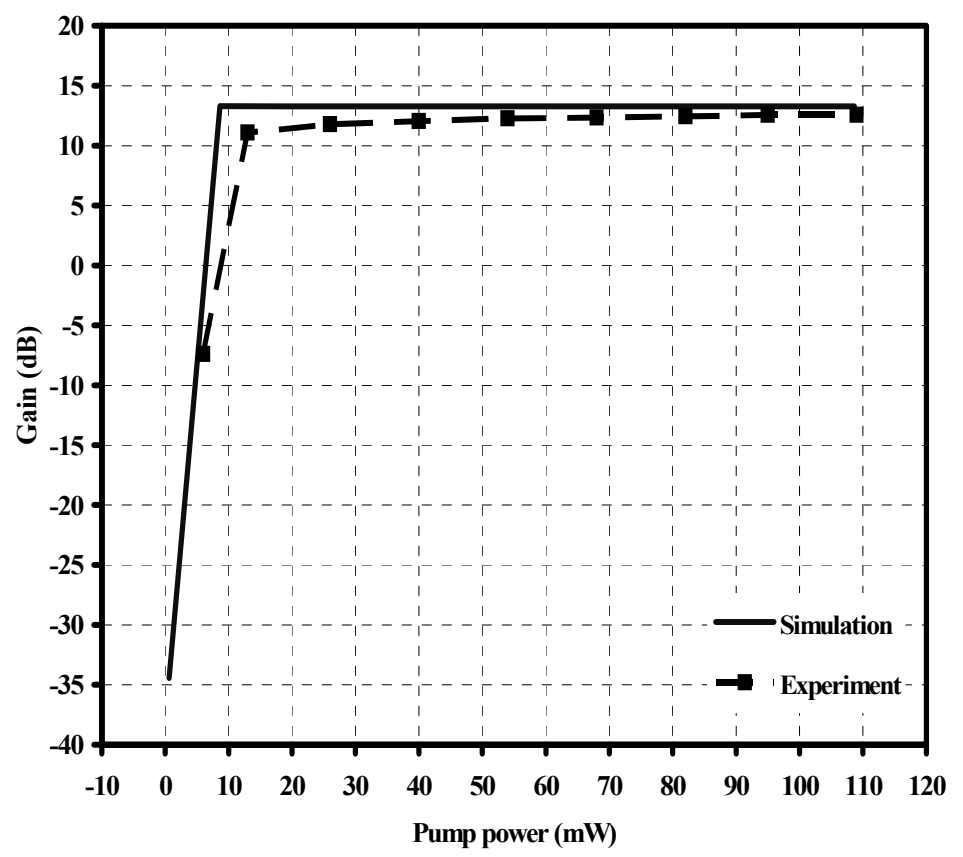

Fig. 2: Comparison between simulated and experimental gain versus pump power profile

The comparison between the experimental and the simulated gain versus pump power profile is shown in Fig. 2 for input signal power of $0 \mathrm{dBm}$. There are two obvious differences between the simulated and the experimental results. First, the pump power value indicating the onset of the laser is different. The experimental results indicate that the clamping effect was onset at around $9 \mathrm{~mW}$, whereas the simulated results indicates that the clamping effect was onset at higher pump power around $14 \mathrm{~mW}$. Second, the simulated results are slightly higher than the actual experimental results.

It is also important to note that the simulated values show ideal clamping effect with absolutely no gain variation in the clamped region whereas, the actual experimental values shows a slight increase as the pump power increases. The experimental clamped gain value is measured to be lower than the simulated clamped gain value by $2.2 \mathrm{~dB}$. This difference is reduced to $0.7 \mathrm{~dB}$ at large input signal power showing a subtle gain increment of $1.5 \mathrm{~dB}$ over the $13 \mathrm{~mW}$ to $109 \mathrm{~mW}$ pump power range.

Comparison between the experimental gain versus input signal profile and the simulated profile shown in Fig. 3 also shows the non-idealistic behaviour of experimental gain. A difference of $0.7 \mathrm{~dB}$ is observed for input signal power of $-40 \mathrm{dBm}$ and it increases as the input signal power increases. At $0 \mathrm{dBm}$, the difference between the experimental and the simulated gain reach up to $1.2 \mathrm{~dB}$. The actual experimental clamped gain values however, show a slight gain decrement of $0.5 \mathrm{~dB}$ as the input signal power was increased from -40 $\mathrm{dBm}$ to $0 \mathrm{dBm}$. On the other hand, the simulated values show ideal clamping effect, where the gain is fixed at $13.3 \mathrm{~dB}$ throughout the whole range of the input signal power of up to $0 \mathrm{dBm}$. 


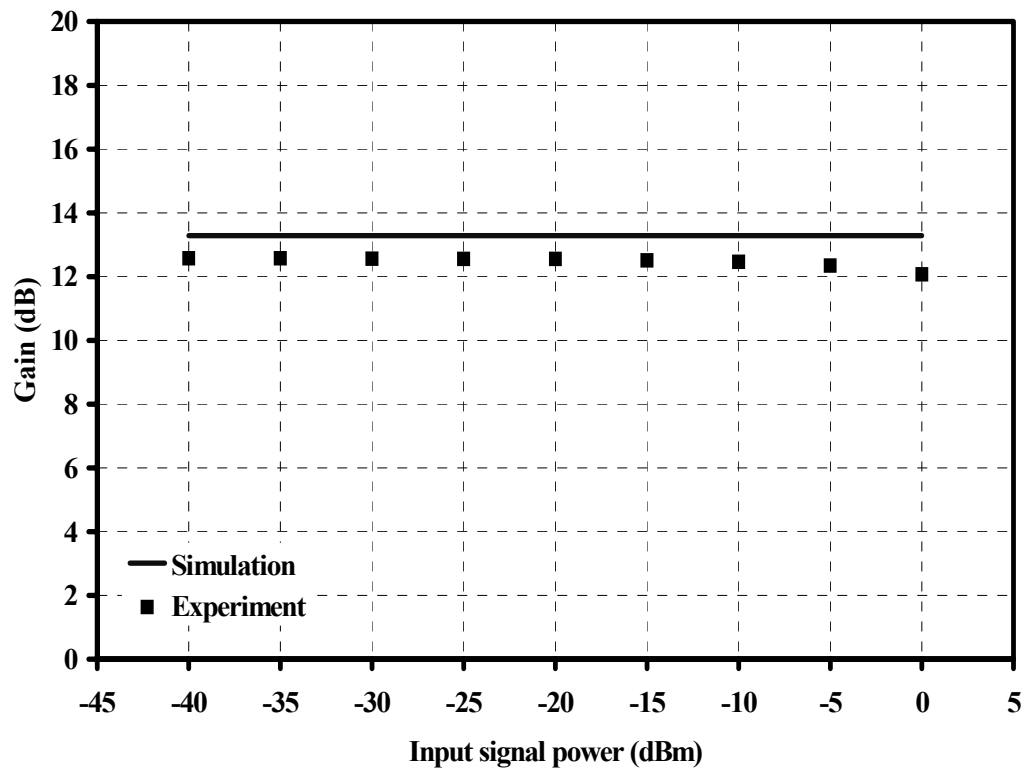

Fig. 3: Comparison between simulated and experimental gain versus input signal power profile

\section{B. GC-EDFA Noise Figure Performance}

The similarity between the measured noise figure values as well as the simulated noise figure profiles against the pump power is shown in Fig. 4. The noise figure differences observed between the experimental and simulated values does not show significant variation across the various pump power values. However, the experimental noise figure value of around $5.5 \mathrm{~dB}$ is slightly lower than the simulated noise figure value, which is about $5.9 \mathrm{~dB}$. This is caused by the difference between the cavity loss in the simulation and the experimental set-up as mentioned earlier.

Figure 5 shows that the $0.4 \mathrm{~dB}$ difference in the experimental and simulated noise figure value at input signal power of $-40 \mathrm{dBm}$ remains the same for input signal power of up to $-15 \mathrm{dBm}$. The experimental noise figure for input signal power beyond $-15 \mathrm{dBm}$ shows an exponential growth, whereas the simulated noise figure remains constant throughout this region. The exponential noise figure growth in the experimental values occurs due to the source spontaneous emission from the signal source. The noise figure at $0 \mathrm{dBm}$ reaches up to a maximum value of $9.2 \mathrm{~dB}$. The signal source in the simulation software is an ideal source, thus there is no source spontaneous source that can degrade to noise figure. The simulated noise figure values remains constant right up to $-5 \mathrm{dBm}$ input signal power. 


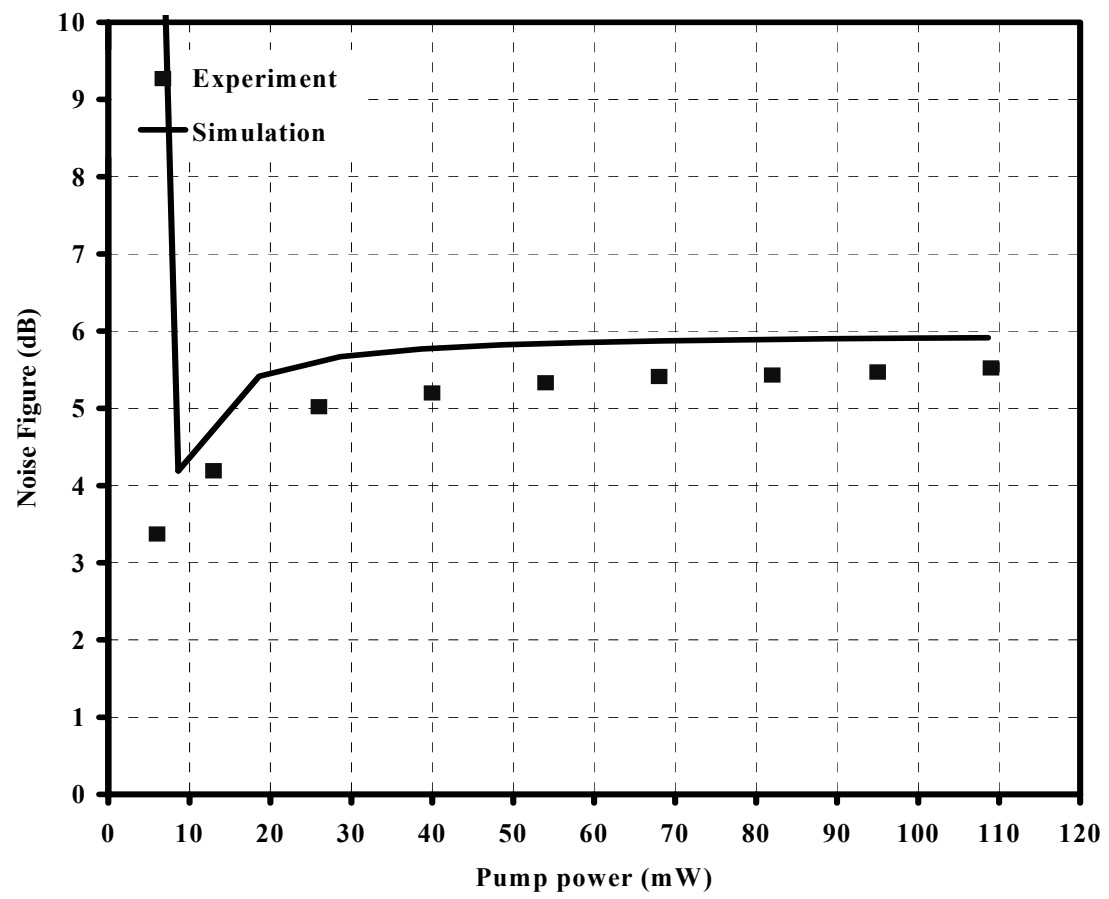

Fig. 4: Comparison between simulated and experimental noise figure versus pump power profile

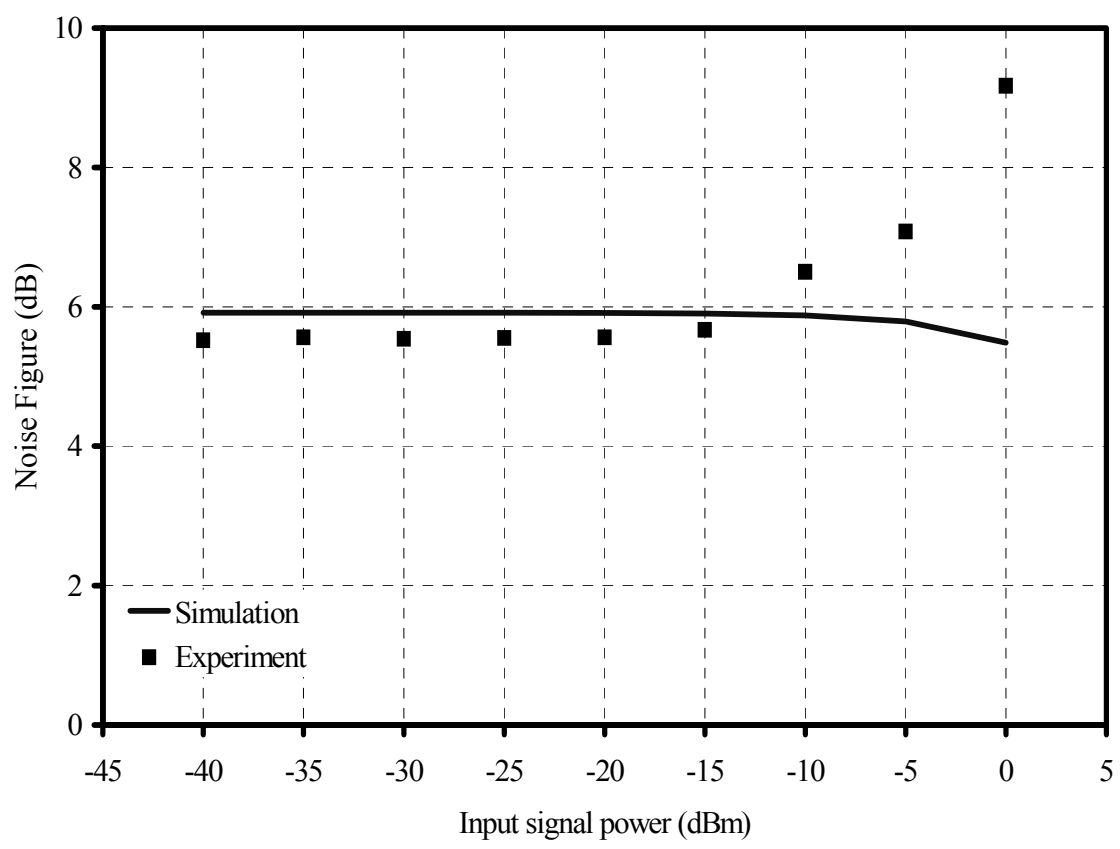

Fig. 5: Comparison between simulated and experimental values noise figure versus input signal power profile 


\section{GC-EDFA Gain Spectrum}

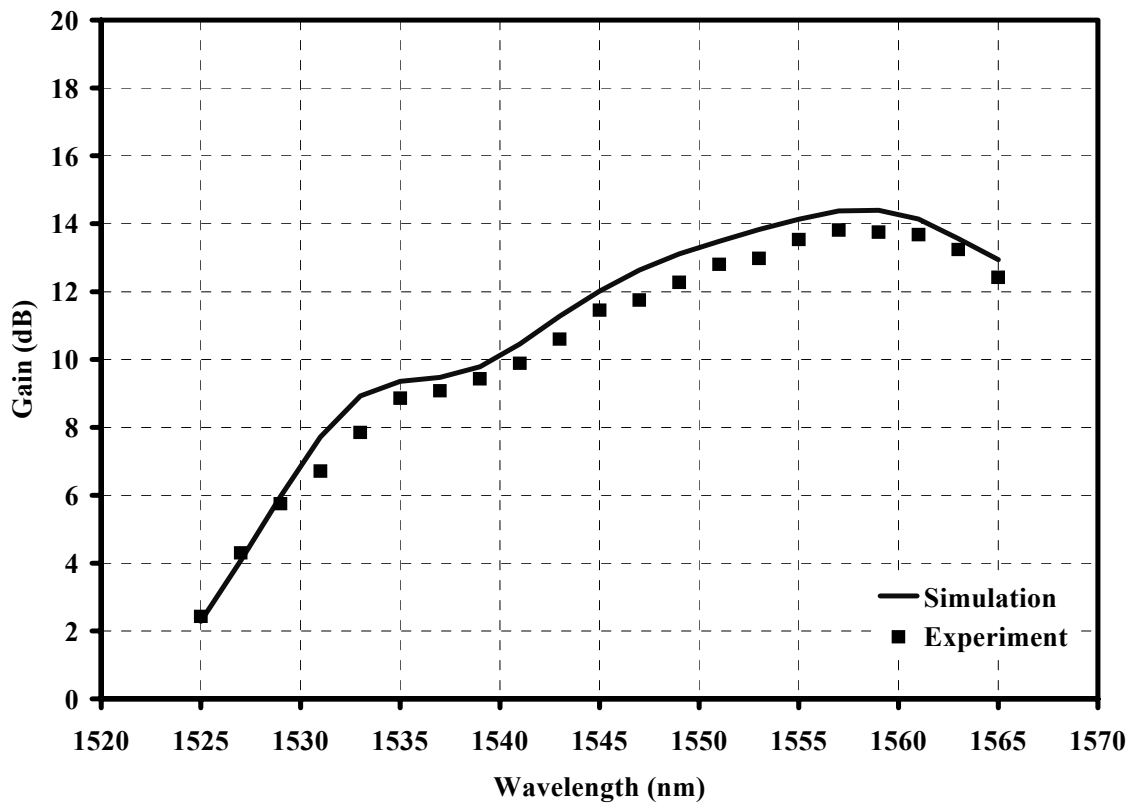

Fig. 6: Comparison between simulated and experimental gain spectrum

The gain spectrum of the experimental values as well as of the simulated values for input signal power of $-40 \mathrm{dBm}$ and pump power $109 \mathrm{~mW}$ is shown to be almost identical by Fig. 6. Although discrepancies between the experimental and the simulated values do exist, the differences observed is very small (less than $1 \mathrm{~dB}$ ) and it varies as the signal wavelength changes. The difference variation across the signal wavelength is small.

\section{GC-EDFA Output Profile}

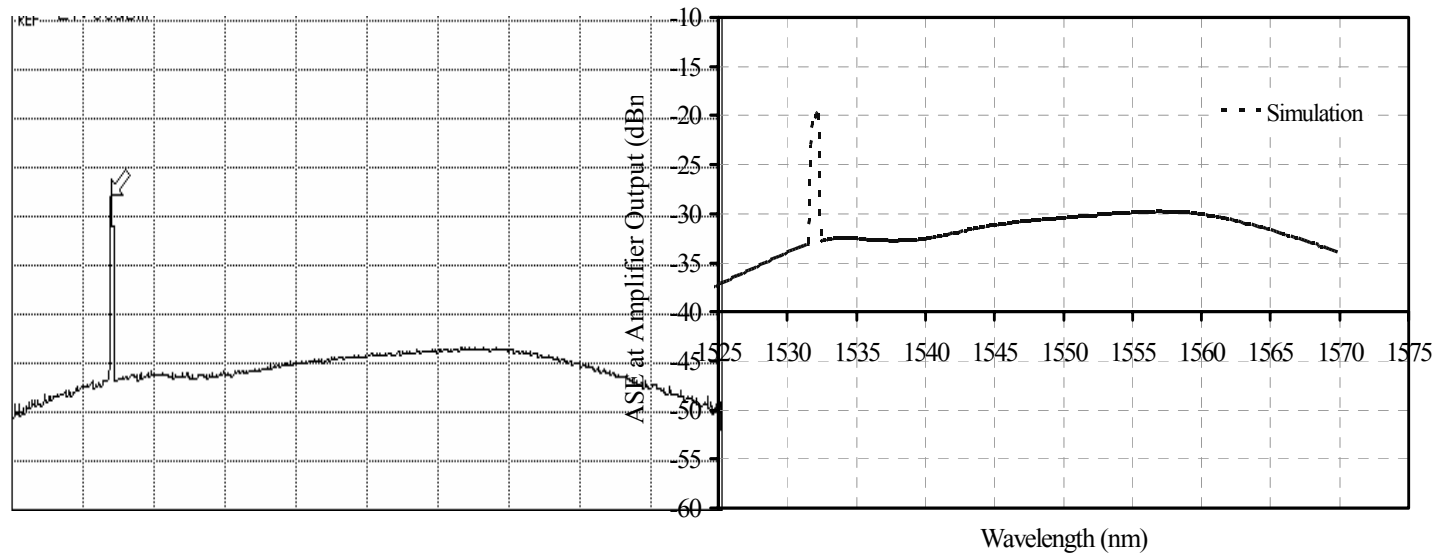

Wavelength (nm)

Experiment

Simulation

Fig. 7: Comparison between simulated and experimental amplifier output 
The GC-EDFA output profile measured and the GC-EDFA output spectrum simulated at pump power of $109 \mathrm{~mW}$ shown in Fig. 7 is almost identical. The $13 \mathrm{~dB}$ difference between the simulated and the experimental ASE levels is similar to the observations for standard EDFA. This however, does not cause significant error in the measurement of the GC-EDFA characteristic values. Both the results show that the back-reflections in the system prevent total elimination of the laser at the output. The laser power level at the output is different, similar to the forward ASE results.

\section{CONCLUSION}

The above comparisons show that the EDFA_Design software version 2.0 simulates the laser controlled counter-propagating GC-EDFA system with high accuracy. This means that the software can be used to simulate various GC-EDFA designs and to design an optimised GC-EDFA system. Simulations of the system gain as well as the noise figure values depict excellent consistency with experimental results. The only obvious discrepancy between the experimental and the simulated results is shown by the noise figure versus input signal power profile in the region of the large input signal power, which however, can be compensated by incorporating the influence of the signal to noise ratio (SNR) degradation of the signal for the large signal power values. Comparison of the experimental and simulated gain spectrum also depicts excellent consistency. The change in spectrum as compared to the standard EDFA gain spectrum has also been simulated with high accuracy.

\section{REFERENCES}

[1] M. Zirngibl, "Gain Control in Erbium-Doped Fibre Amplifiers by an All-Optical Feedback Loop,” Electron. Lett., Vol. 27, No. 7, pp. 560 - 561, March 1991.

[2] E. Desurvire, "Erbium-Doped Fiber Amplifiers: Principles and Applications," John Wiley \& Sons, Canada, p. vi, preface, p. 421, chapter 5, p. 469, chapter 6, 1994.

[3] D.R. Hall, and P.E. Jackson, "The Physics and Technology of Laser Resonator," p. 62, chapter 4, 19.

[4] M. Kobayashi, and S. Muro, "Gain Stabilisation in Erbium Doped Fiber Amplifier with Optical Feedback Loop Using Circulators," in Tech. Dig. OECC '98, pp. 98 - 99, 1998.

[5] M.M. Kozak, R. Caspary, and U.B. Unrau, "Computer Aided EDFA Design, Simulation and Optimization," Proc. of 3rd Int. Conf. on Transparent Optical Networks 2001, pp. 202 - 204, 2001. 